\title{
Protocol
}

\section{The point prevalence of post-partum perinatal mental health disorders and associated psychosocial characteristics in Malta: a study protocol}

\author{
Rachel Buhagiar*, Kristina Bettenzana
}

Department of Psychology, University of Malta, Msida, Malta

Received: 05 January 2022

Revised: 19 January 2022

Accepted: 20 January 2022

\author{
*Correspondence: \\ Dr. Rachel Buhagiar, \\ E-mail: rachel.buhagiar.07@um.edu.mt
}

Copyright: () the author(s), publisher and licensee Medip Academy. This is an open-access article distributed under the terms of the Creative Commons Attribution Non-Commercial License, which permits unrestricted non-commercial use, distribution, and reproduction in any medium, provided the original work is properly cited.

\begin{abstract}
Background: Perinatal mental health disorders are considered to be a major public health concern, affecting approximately $20 \%$ of pregnant and postnatal women. Left untreated, maternal psychopathology can extent to the rest of the family unit, including the child. Thus, a better understanding of the overall burden of these disorders is fundamental to inform policy makers and produce practice change. To-date, the exact prevalence of these disorders in Malta, an island in the center of the Mediterranean Sea, remains unknown. The primary objective of this crosssectional study will be to address this gap in knowledge and define the local prevalence of perinatal psychopathology. Methods: A representative sample of approximately 250 postnatal women from birth up to one year after childbirth, resident in Malta, will be recruited through a stratified random technique. Participation will involve two phases. In the first stage, selected participants will be asked to self-complete the Edinburgh Postnatal Depression Scale (EPDS), the Generalised Anxiety Disorder-7 item (GAD-7), the Yale Brown Obsessive Compulsive Scale (YBoCS), the PostTraumatic Stress Disorder Checklist for DSM-V (PCL-5), and the Difficulties in Emotional Regulation Scale (DERS) questionnaires. In the second stage, those women who score equal or above the cut-off values in any of these questionnaires, will be assessed using the diagnostic Mini-International Neuropsychiatric Interview (MINI) to confirm or refute a diagnosis of a mental health disorder. Basic demographic details and psychosocial characteristics will be recorded.
\end{abstract}

Conclusions: This study will help determine the point prevalence of postnatal perinatal psychopathology and associated psychosocial determinants in Malta.

Keywords: Perinatal mental health disorders, Perinatal psychopathology, Postpartum, Prevalence

\section{INTRODUCTION}

The perinatal period and maternal mental health disorders

The perinatal period, as defined in relation to mental illness, includes the time of conception up to when the child reaches the age of one. Perinatal psychiatric disorders cover a wide range of conditions, and may include, but are not limited to depression, anxiety, obsessive-compulsive disorder and post-traumatic stress disorder. ${ }^{1}$ These illnesses may arise for the first time in pregnancy or after childbirth, but they can also be present pre-conceptionally and persist in the perinatal period.

Globally, perinatal mental health problems are considered to be a major public health concern. ${ }^{2}$ Left untreated, perinatal mental health disorders can be associated with significant maternal morbidity and mortality, and can have a lasting deleterious effect on the entire family unit. ${ }^{2,3}$ As evidence from the MBRRACE-UK confidential enquiry into maternal deaths, maternal 
suicide was identified as the third largest cause of direct maternal death in the first weeks after birth, and the leading cause of death when looked at over a year. ${ }^{4}$ Furthermore, the economic costs associated with perinatal mental health disorders equate to approximately $£ 10,000$ for each single birth. ${ }^{5}$ This latter study identified that $72 \%$ of these costs related to adverse child outcomes, for example, emotional and conduct problems, special educational needs and leaving school without qualifications. Maternal psychopathology has in fact been linked with increased likelihood of psychological and psychiatric co-morbidities in the offspring. ${ }^{6,7}$ This emphasizes the importance of early recognition and treatment of maternal mental health difficulties to prevent these long-term intergenerational negative sequelae.

\section{The prevalence of perinatal mental health disorders}

The prevalence rates of perinatal mental health illnesses varies between different studies and geographical areas. Worldwide, between $10 \%$ and $20 \%$ of women are known to experience a mental disorder in pregnancy and after birth..$^{5}$ There is some indirect evidence that the prevalence of these disorders has increased in recent years, as shown in the higher proportion of children being exposed to maternal mental illness. ${ }^{8}$ Furthermore, during these unprecedented times of the COVID-19 pandemic, an increased rate of mental health difficulties in perinatal women is being reported in the literature. ${ }^{9,10}$

In a systematic review and meta-analysis which included 58 articles, the overall prevalence of postpartum depression was identified to be $17 \%$ (95\% CI 0.15-0.20), with a statistically significant difference between different geographical regions. Europe was found to have the lowest rate $(8 \%, 95 \%$ CI $0.05-0.11)$. No difference in prevalence rate was found among mothers with and without a previous depressive history. Interestingly, an increasing prevalence was observed beyond six months postnatally. ${ }^{11}$ For postnatal anxiety disorder, a prevalence of $10 \%$ was identified, with a similar difference rate between low, medium and highincome countries. $^{12}$

To-date, there is paucity of literature data on the prevalence of postnatal mental health disorders in Malta. Malta is the largest of the three Maltese islands, located right in the middle of the Mediterranean. Despite its small area, the Maltese archipelago is quite dense, with approximately 475,000 inhabitants, and around 4000 births annually. ${ }^{13}$ In 2004, a local study identified the point prevalence of postnatal depression to be $8.7 \% .{ }^{14}$ To-date, no other prevalence studies have been conducted.

In the context of these limitations, the main objective of this research will be to determine the point prevalence of some of the commonest postpartum psychiatric disorders in Malta, such as depression, anxiety and obsessivecompulsive disorder. A secondary objective will be to obtain a cut-off point value for a provisional diagnosis for commonly used self-report mental health screening questionnaires for the perinatal population in Malta.

\section{METHODS}

\section{Study population and sampling methods}

Participating mothers need to be 18 years and above, within 12 months postnatally, able to read and understand the Maltese and/or English language, and give written consent. Excluded from the study will be mothers who are unable to consent to participation.

In this study, stratified random sampling will be used to ensure a representative sample of the population of interest. In 2018, there were a total of 4439 registered live births in Malta. ${ }^{15}$ Based on international prevalence data, it is estimated that between 665 and 887 of mothers in Malta will suffer from ill-mental health postnatally. This constitutes our population of interest. The calculated sample size will be of approximately 269 postnatal women $(95 \% \mathrm{CI}$; $5 \%$ margin of error). To account for non-response, a total sample of 300 women will be recruited.

In 2018, the national statistics office (NSO) ${ }^{13}$ reported a variation in live births across the different regions and districts in the Maltese Islands, with the highest and lowest rate being in Northern Harbour (27\%) and Gozo and Comino (7\%) respectively. Also, the number of deliveries varied according to maternal age. ${ }^{15}$ The greatest number of deliveries $(37.3 \%)$ occurred in the age group of 20 to 34 years. Given this reported variation, regional districts and maternal age will be used as our two main strata or subgroups to then complete the sampling process. A calculated proportionate sampling will then follow for each sub-group based on the reported birth percentages for each to achieve a total sample size of 300 women. In other words, the sample size of each stratum will be equal to the subgroup's proportion in the postnatal population in Malta as a whole. Simple random sampling using computer generated lists will then be used to identify participants from each of these groups to be included in the study.

\section{Processes and statistical analysis}

Postnatal mothers, resident in Malta, of any nationality, who have given birth between November 2020 and November 2021 at Mater Dei hospital, the main general hospital in Malta, will be randomly selected using the process described above and invited to participate in the study. One limitation of this research is that women who have delivered in the private sector will not be accounted for in this study. According to the national obstetric information system (NOIS) $)^{15}, 0.8 \%$ and $0.5 \%$ of deliveries occurred within private institutions in 2018 and 2019, respectively. Hence, these women represent only a minority of the perinatal population in Malta. 
Postnatal mothers identified through the stratified and simple random sampling will be sent a research pack at their home address, consisting of a welcome letter, an information leaflet about the project and their participation, and a consent form. They will be provided with contact details of the main researcher in case they would like to get in touch to discuss further. The research pack will be sent by an intermediary to abide with institutional data protection guidelines.

If the mother then agrees to participate, she will be asked to sign the included consent form and to send it back to the research team in the self-addressed envelope. This does not preclude them from withdrawing from the study at any stage, without suffering any repercussions or implications. The participants will also be asked to complete a number of self-report questionnaires about her mental health, including the Edinburgh Postnatal Depression Scale (EPDS), the Generalized Anxiety Disorder-7 item (GAD-7), the Yale Brown Obsessive Compulsive Scale (YBoCS), the Post-Traumatic Stress Disorder Checklist for DSM-V (PCL-5), and the Difficulties in Emotional Regulation Scale (DERS). These scales which assess for symptoms of postnatal depression, generalised anxiety, obsessive-compulsive, post-traumatic stress disorder and emotion problems respectively, are recommended as screening measures in the perinatal period by the Royal College of Psychiatrists. ${ }^{16}$ These instruments are all readily available and accessible online.

For those instruments not available in the Maltese language, the original English version will be translated into Maltese, back-translated and cultural adapted to the Maltese perinatal population. This will be followed by a test-retest reliability assessment. In this way, participants can be provided with both language versions of the questionnaires.

These five questionnaires which take a total of approximately 15 to 20 minutes to be completed, can then be returned to the research team by post or email. Participants will also be given the choice to complete the questionnaires online by accessing a QR code, with the aim of increasing response rate. Moreover, if no response is achieved after two weeks, a postal reminder will be sent to selected subjects. ${ }^{17}$ Another measure to increase participation will be to automatically include respondents who return the questionnaires in a ballot for non-monetary incentives. ${ }^{18}$

Once received, returned questionnaires will be scored by the researcher. Participants who score equal or above the cut off scores for the following individual scales will be invited for a diagnostic assessment as highlighted below. The selected cut off values include the following: 10 or above in the EPDS and/or GAD, 8 or above in the YBoCS, 36 or above in the PCL-5, and 80 or above in the DERS. A lower cut-off value than the one recommended in the literature for each of these instruments will be used for the purpose of this study to avoid false negatives and to enhance detection of participants who meet diagnostic criteria. 19-22 These participants will then be assessed using the Mini International Neuropsychiatric Interview (MINI), a semi-structured instrument to confirm or refute a diagnosis of a mental health disorder, its nature and severity. ${ }^{23}$ This interview which will be conducted within 2 weeks of receiving the self-report questionnaires, will be carried out by an independent and trained mental health professional with significant experience in the field, at a time and place which is convenient for the participant. This assessment may also be carried remotely if the participant prefers. The prevalence will then be calculated as a measure of frequency. ${ }^{24}$

Basic demographic details and characteristics, such as maternal and infant age, relationship status, educational level and employment, number of pregnancies and other children in the network, will be recorded. Information about the mother's medical, psychiatric and family history will also be obtained, as well as details about medications.

The scores of the self-report symptom scales will be correlated using the receiver operating characteristic curve (ROC) to the MINI diagnostic outcomes to calculate the best cut-off point for a provisional diagnosis for each of these scales when applied in the Maltese perinatal population.

\section{DISCUSSION}

\section{Potential implications}

This study will be the first in several years to provide an assessment of the overall burden of postpartum perinatal mental health disorders in Malta, and therefore, it will be of interest to policy makers in determining whether there is need for further investment in this field. Furthermore, given the early recognition of perinatal sufferers and their referral to specialist treatment, this will benefit individual women and their families by minimizing the risk of adverse effects.

Another benefit is that in learning about individual selfreport instruments and their cut-off value for a provisional diagnosis, they can be subsequently implemented within local healthcare settings for screening of women for perinatal mental health pathology. These questionnaires have the advantage that they are brief and can be completed within a short time frame, for example whilst clients are waiting to be seen by the clinician. Whilst such measures cannot replace formal assessments to confirm or refute a diagnosis, their implementation within local services can be a valuable aid to clinicians in their everyday diagnostic assessments and busy schedules.

\section{Strengths and limitations}

Symptom scales compared to diagnostic interview are known to yield significantly higher prevalence rates. ${ }^{25}$ 
To address this issue, the point prevalence will not be based solely on self-report measures, but a diagnostic interview will be utilized. Furthermore, whilst all the self-report instruments selected for this study are recommended by the Royal College of Psychiatrists and are widely used in the general adult and perinatal populations, not all of them have been specifically validated for use in the pregnant and postnatal women, or more specifically in the Maltese population. ${ }^{16}$ To-date, for the Maltese perinatal population, validation is only available for the EPDS and PCL-5. ${ }^{26,27}$ The validation for the remaining questionnaires will be available on completion of this project.

This study will not account for the small proportion of women who choose to deliver in the private sector, However, given that these women represent a minority of the perinatal population in Malta, the sample of this research will still be representative of the population of interest, and therefore, this will not impact on the accuracy of data. Another weakness is that no control group will be used to compare the prevalence of mental health disorders in women who have not given birth. Moreover, whilst the perinatal period constitutes both pregnancy and the first year after childbirth, this study will focus solely on the postnatal period. Further subsequent studies will be needed to determine the prevalence of antenatal mental health issues for a 'total' local prevalence rate of perinatal disorders.

\section{CONCLUSION}

This cross-sectional study will address the existing gap in knowledge and provide a prevalence rate of perinatal mental health disorders in postpartum women in Malta. A better understanding of the local burden of these disorders will help guide the development of specialist perinatal services for the benefit on families and society in general.

Funding: Funding sources by the Tertiary Education Scholarship Scheme from the Ministry of Education in Malta.

Conflict of interest: None declared

Ethical approval: The study was approved by the Institutional Ethics Committee

\section{REFERENCES}

1. O'Hara MW, Wisner KL, Asher A. Perinatal mental illness: definition, description and aetiology. Best Pract Res Clin Obstet Gynaecol. 2014;28:3-12.

2. NHS England. Perinatal, 2019. Available at: https://www.england.nhs.uk/mental-health/perinatal/. Accessed on 4 May 2021.

3. Myors KA, Schmied V, Johnson M, Cleary M. Therapeutic interventions in perinatal and infant mental health services: a mixed methods inquiry. Issues Ment Health Nurs. 2014;35:372-85.
4. Nuffield department of Population Health (NPEU). Confidential enquiry into maternal death, 2017. Available at: https://www.npeu.ox.ac.uk/mbrraceuk/reports/confidential-enquiry-into-maternal ondeaths. Accessed 25 April 2021.

5. Bauer A, Parsonage M, Knapp M, Iemmi V, Adelaja B. The costs of perinatal mental health problems, 2014. Available at: https://www.nwcscnsenate. nhs.uk/files/3914/7030/1256/Costs_of_perinatal_mh. pdf. Accessed on 25 April 2021.

6. Leadsom A, Field F, Burstow P, Lucas C. The 1001 critical days. The importance of the conception to age two period, 2019. Available at: http://anepeducacionprenatal.org/wp-content/ uploads/2018/02/The_1001_Critical_Days_Manifest o.pdf. Accessed on 5 May 2021.

7. Government of South Australia. South Australian perinatal practice guideline. Assessing parent infant relationship, 2018. Available at: https://www. sahealth.sa.gov.au/wps/wcm/connect/1d2582004ee1 d8d1ac7badd150ce4f37/Assessing+parent+infant+rel ationship_PPG_v2_0.pdf?MOD=AJPERES\&CACH EID=ROOTWORKSPACE-

1d2582004ee1d8d1ac7badd150ce4f37-mOYgEVG. Accessed on 10 May 2021.

8. Howard LM, Khalifeh H. Perinatal mental health: a review of progress and challenges. World Psychiatry. 2020;19:313-27.

9. Chmielewska B, Barratt I, Townsend R, Kalafat E, van der Meulen J, Gurol-Urganci I et al. Effects of the COVID-19 pandemic on maternal and perinatal outcomes: a systematic review and metaanalysis. The Lancet. 2021; 9(6):e759-72.

10. Racine, N, Hetherington E, McArthur BA, McDonald S, Edwards S, Tough S et al. Maternal depressive and anxiety symptoms before and during the COVID-19 pandemic in Canada: a longitudinal analysis. The Lancet 2021;8(5):405-15.

11. Shorey S, Chee CYI, Ng ED, Chan YH, Tam WWS, Chong YS. Prevalence and incidence of postpartum depression among healthy mothers: a systematic review and meta-analysis. J Psychiatr Res. 2018;104:235-48.

12. Dennis C-L, Falah-Hassani K, Shiri R. Prevalence of antenatal and postnatal anxiety: systematic review and meta-analysis. Br J Psych. 2017;210:315-23.

13. National Statistics Office, Malta. Regional Statistics Malta-2020 Edition, 2020. Available from https://nso.gov.mt/en/publicatons/Publications_by_U nit/Documents/02_Regional_Statistics_(Gozo_Offic e)/2020/Regional_Statistics_Malta-

2020\%20Edition.pdf. Accessed on 10 June 2021.

14. Felice E, Saliba J, Grech V, Cox J. Prevalence rates and psychosocial characteristics associated with depression in pregnancy and postpartum in Maltese women. J Affect Disord. 2004;82:297-301.

15. Gatt M, Zahra C. National Obstetrics Information System, Directorate for Health Information and Research. NOIS Annual Report, 2019. Available at: 
https://deputyprimeminister.gov.mt/en/dhir/Pages/Re gistries/births.aspx. Accessed on 25 April 2021.

16. The Royal College of Psychiatrists. Framework for routine outcome measures in perinatal psychiatry, 2018. Available at: https://www.rcpsych.ac.uk/ docs/default-source/improving-care/better-mhpolicy/college-reports/college-report-cr216.pdf? sfvrsn=12b1e81c_2. Accessed on 10 June 2021.

17. Harrison S, Henderson J, Alderdice F, Quigley MA. Methods to increase response rates to a populationbased maternity survey: a comparison of two pilot studies. BMC Med Res Methodol 2019;19:1-8.

18. Toepoel V, Schonlau M. Dealing with nonresponse: strategies to increase participation and methods for postsurvey adjustments. Mathl Popul Stud 2017;24:79-83.

19. Levis B, Negeri Z, Sun Y, Benedetti A, Thombs BD. Accuracy of the Edinburgh Postnatal Depression Scale (EPDS) for screening to detect major depression among pregnant and postpartum women: a systematic review and meta-analysis of individual participant data. BMJ. 2020:1-11.

20. Centre of Perinatal Excellence (COPE). Australian Perinatal Mental Health Guideline Evidence Review. Technical report part B. Psychosocial assessment and screening for depression or anxiety, 2017. Available from https://www.cope.org.au/health-professionals/ health-professionals-3/review-of-new-perinatalmental-health-guidelines/. Accessed on 5 May 2021.

21. Storch EA, De Nadai A, Conceicao do Rosario M, Shavitt RG, Torres AR, Ferrao YA et al. Defining clinical severity in adults with obsessive-compulsive disorder. Compr Psychiatry, 2015;63:30-35.

22. Connell J, Barkham M, Stiles WB, Twigg E, Singleton N, Evans O, Miles J. Distribution of CORE-OM scores in a general population, clinical cut-off points and comparison with the CIS-R. BJPsych. 2007;190:69-74.

23. Sheehan DV, Lecrubier Y, Sheehan KH, Amorim P, Janavs J, Weiller E et al. The Mini-International Neuropsychiatric Interview (M.I.N.I): the development and validation of a structured diagnostic psychiatric interview for DSM-IV and ICD-10. J Clin Psychiatry. 1998;20:22-33.

24. Ressing M, Blettner M, Klug SJ. Data analysis of epidemiological studies. Dtsch Arztebl Int. 2010;107:187092.

25. Woody CA, Ferrari AJ, Siskind DJ, Whiteford HA, Harris MG. A systematic review and meta-regression of the prevalence and incidence of perinatal depression. J Affect Disord. 2017;219:86-92.

26. Felice E, Saliba J, Grech V, Cox J. Validation of the Maltese version of the Edinburgh Postnaatl Depression Scale. Arch Women Ment Health. 2006;9:75-80.

27. Buhagiar R, Dimech C, Felice E. Validation of the Post-Traumatic Stress Disorder Checklist for DSM-5 in the Maltese Perinatal Population. MMJ. 2021.

Cite this article as: Buhagiar R, Bettenzana $\mathrm{K}$. The point prevalence of post-partum perinatal mental health disorders and associated psychosocial characteristics in Malta: a study protocol. Int J Clin Trials 2022;9(1):459. 